\title{
The Usage of the Digital Controller in Regulating Boost Converter
}

\author{
Muhanad D. Almawlawe, Darko Mitic, Marko Milojevic \\ Department of Control Systems, Faculty of Electronic Engineering, University of Niš, Niš, Republic of Serbia \\ Email: muhanadhashim@gmail.com,darko.mitic@elfak.ni.ac.rs, marko.milojevic@elfak.ni.ac.rs
}

Received 22 March 2015; accepted 6 December 2015; published 9 December 2015

Copyright (C) 2015 by authors and Scientific Research Publishing Inc.

This work is licensed under the Creative Commons Attribution International License (CC BY). http://creativecommons.org/licenses/by/4.0/

cc) (i) Open Access

\begin{abstract}
This paper proposes the modeling and simulation technique to analyze and design a Boost converter using generalized minimum variance method with discrete-time quasi-sliding mode to adjust the converter switch through a pulse width modulation (PWM), so as to enhance a stable output voltage. The control objective is to maintain the sensed output voltage stable, constant and equal to some constant reference voltage (8 volt) in the load resistance variation $(24,48,240) \Omega$ and input voltage variation $(20,24,28)$ volt circumstances. This control strategy is very appropriate for the digitally controlled power converter and for the system requirement accomplishment, resulting high output voltage accuracy. The performance degradation in practical implementation can be expected due to noise, PWM nonlinearities, and components imperfection. The digital simulation using MATHLAB/Simulink is performed to validate the functionality of the system.
\end{abstract}

\section{Keywords}

Boost Converter, Input-Output Measurement, QSM (Quasi-Sliding Mode), GMV (Generalized Minimum Variance), PWM (Pulse Width Modulation)

\section{Introduction}

The DC-DC converters are essential in a variety of applications such as power supplies, personal computers, cellular phones, office equipment's, spacecraft power systems, telecommunications equipment's, and DC motor starting circuits, where input/output voltage ranges overlap; the DC-DC converters convert a DC input voltage $V_{i}(t)$, to a DC output voltage $V_{o}(t)$, with a magnitude other than the input voltage [1]. According to the electrical components combination and their connection to each other in different ways, different topologies are formed, with different properties: The Buck, Boost, and Buck-Boost converters are three basic converter topologies; also 
there are different types of conversion method such as electronic, linear, switched mode, magnetic, and capacitive. The circuits described in this paper are classified as switched mode DC-DC Boost converter [2] [3]. Boost converter is a type of DC-DC converters that have an output voltage magnitude which is greater than the input voltage magnitude. These DC-DC converters are required to produce a regulated output voltage, by storing the input energy temporarily and then releasing this energy to the output at a different voltage. The storage may be in magnetic field; this kind of regulation is powerful than the linear voltage regulation which dissipates unwanted power as heat which may cause difficulty in cooling resulting degrade in efficiency. The topology under discussion is Boost converter which has an output voltage magnitude $48 \mathrm{v}$ that is higher than the input voltage magnitude $(20,24,28) \mathrm{v}$.

As it is known, sliding mode (SM) control provides robust system motion along a predefined sliding surface due to its insensitivity to parameter uncertainty and external disturbance under certain conditions [4], which is adequate for power converting systems under load variation [5]. By using pulse width modulation (PWM) we achieve the limitation of the switching frequency in order to make the SM controllers more applicable [6] [7].

The quasi-sliding mode controller can assure fixed switching frequency, which is held by discretization of the sliding mode control which can induce undesirable chattering in the vicinity of sliding surface [8]. A combination of the generalized minimum variance and discrete-time sliding mode control, results in the quasi-sliding mode control algorithm [9]. This method can be used in the voltage control of the Boost converter considered in this paper which is based only on the output voltage measurement, resulting in better system accuracy in steady state, and provides chattering alleviation.

In this paper, the second section considers the system modeling and the evolution of the state space equations of the boost converter, transfer function and the design procedure of discrete-time quasi-sliding mode controller. The third section considers complete model simulation results in MATLAB. The simulations are done in three scenarios to validate the proposed converter, while the fourth section considers a conclusion dealing with this paper.

\section{DC-DC Boost Converter Modeling and Design}

The Boost converter is a converter which its output voltage magnitude is greater than the input voltage magnitude, it is a typical DC-DC switching converter normally used as a power supply in a wide variety of applications, In modeling of the state space, the state variable which principally are the elements that store the energy of circuit or system (capacitance voltage and inductor current) have significant importance. In an electronic circuit, the first step in modeling is converting the complicated circuit, into basic circuit in which the circuit lows can be established, we will consider that all the elements are ideals in our modeling [2] [3].

The schematic diagram of the Boost converter with quasi-sliding mode controller is shown in Figure 1, During normal operation of the Boost power stage, the electric switch $S_{W}$ is repeatedly switched on and off with the on- and off-times governed by the control circuit. This switching action gives rise to a train of pulses at the junction of $\mathrm{S}_{\mathrm{W}}, \mathrm{D}$, and $\mathrm{L}$. Although the inductor, $\mathrm{L}$, is connected to the output capacitor, C, only when D conducts, an effective $\mathrm{L} / \mathrm{C}$ output filter is formed.

The sensed output voltage $\beta V_{o}$ with referent voltage $V_{\text {ref }}$ are feed to the quasi-sliding mode controller input, at the output we get $u_{k}$ which is compared with the ramp signal providing a PWM signal $u$ Which drives switch $S_{w}$. As in [10] it is proven that the average dynamic of system with sliding mode control is equivalent to the average dynamic of PWM controlled system, implying that the control of equivalent control approach in sliding mode $u_{e q}$ is equivalent to the duty cycle control signal of PWM controller $\left(0<d=V_{C} / V_{\text {ramp }}<1\right)$ [11].

\subsection{System Modeling Mode}

System modeling is probably the most important phase in any form of system control design work. The choice of a circuit model depends upon the objectives of the simulation. If the goal is to predict the behavior of a circuit before it is built. A good system model provides a designer with valuable information about the system dynamics. The state-space description of the converter model in terms of the desired control variables (i.e., voltage and/or current etc.) is the first step to the design of an SM controller, in this paper the controller under study is a second-order quasi-sliding mode voltage controller operating in CCM. The mathematical model for simulation studies can be easily derived by applying Kirchhoff's laws, From Figure 1 the space-state equation is: 


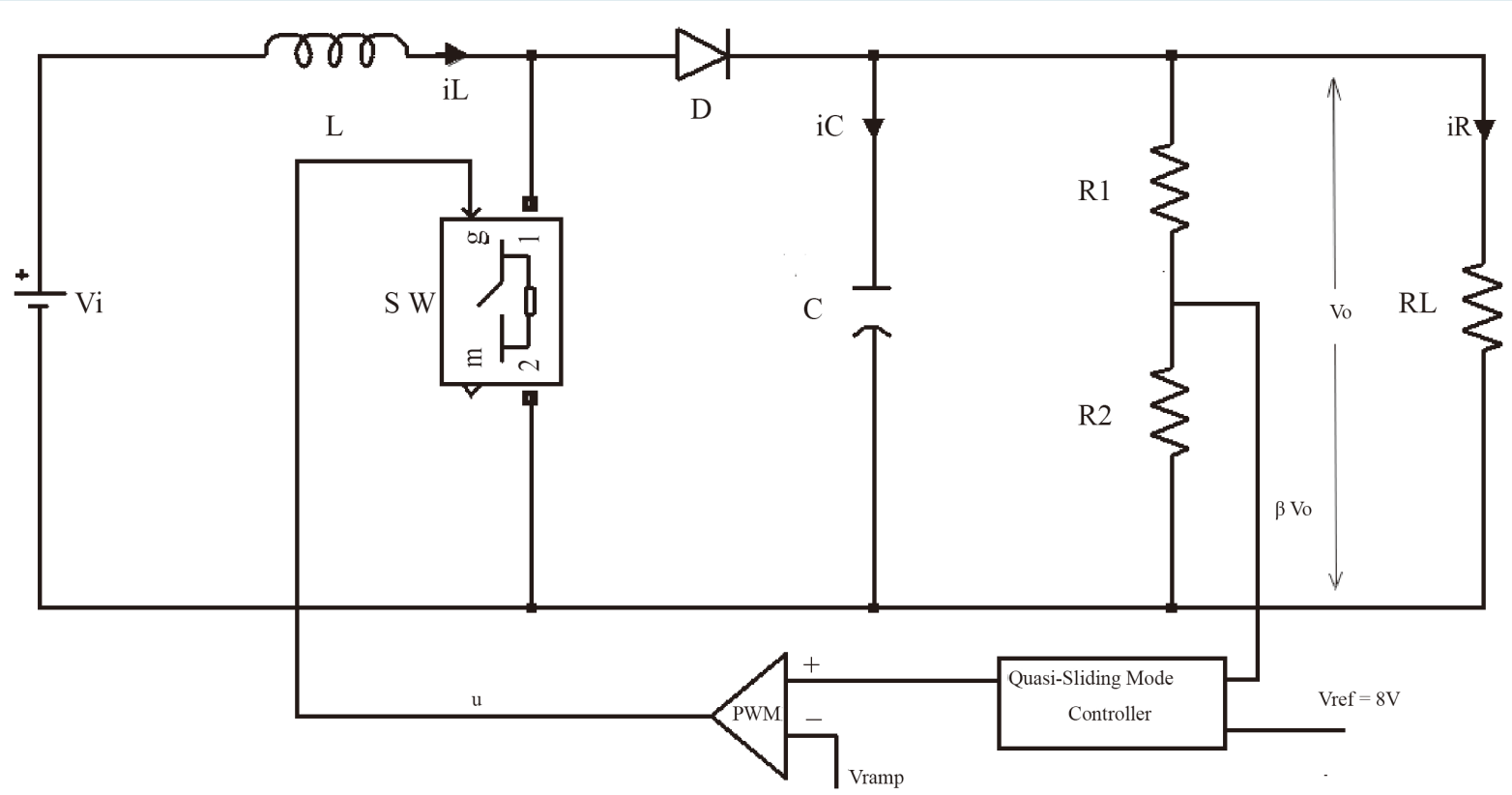

Figure 1. DC-DC Boost converter using quasi-sliding mode controller.

$$
\left[\begin{array}{l}
\dot{x}_{1} \\
\dot{x}_{2}
\end{array}\right]=\left[\begin{array}{cc}
0 & 1 \\
0 & -\frac{1}{R_{L} C}
\end{array}\right]\left[\begin{array}{l}
x_{1} \\
x_{2}
\end{array}\right]+\left[\begin{array}{c}
0 \\
\frac{\beta\left(V_{i}-V_{o}\right)}{L C}
\end{array}\right] \bar{u} .
$$

The state space continuous-time model of Boost converter with of single input-output single is:

$$
\dot{x}(t)=\boldsymbol{A x}(t)+\boldsymbol{B} \bar{u}(t)
$$

where: $x(t)=\left[\begin{array}{llll}x_{1}(t) & x_{2}(t) \cdots x_{n}(t)\end{array}\right]^{\mathrm{T}} \in R^{n}$ state space vector $\bar{u}(t) \in R$ input of the system under control, matrix $\boldsymbol{A}=\left[a_{i j}\right]_{n \times n}, \quad \boldsymbol{B}=\left[b_{i}\right]_{n \times 1}$, in the controllable canonical form is:

$$
\boldsymbol{A}=\left[\begin{array}{cc}
0 & 1 \\
0 & -\frac{1}{R_{L} C}
\end{array}\right], \quad \boldsymbol{B}=\left[\begin{array}{c}
0 \\
\frac{\beta\left(V_{i}-V_{o}\right)}{L C}
\end{array}\right], \quad \boldsymbol{C}=\left[\begin{array}{ll}
1 & 0
\end{array}\right] .
$$

Here $C, L, R_{L}$ denote the capacitance, inductance, and instantaneous load resistance of the converters respectively; $i_{c}, i_{L}, i_{r}$ denote the instantaneous capacitor, inductor, and load currents, respectively; $v_{r e f}, v_{i}, v_{0}$ denote the reference, instantaneous input, and instantaneous output voltages, respectively; $\beta$ denotes sensor gain; $\bar{u}$ is the inverse logic of, $u(t)$ is 0 or 1 , representing the switching state of power switch.

As $u(t)$ is equal to PWM output, which corresponds to equivalent control $u_{\text {equ }}$ in sliding mode control theory, we will assume that $u(t)$ is the quasi-sliding mode controller output [11]-[14]. From (3), the transfer function of the DC-DC Boost converter can be written as:

$$
\begin{gathered}
W_{\text {Boost }}(s)=\boldsymbol{C}(s I-\boldsymbol{A})^{-1} \boldsymbol{B} \\
W_{\text {Boost }}(s)=\frac{Y(s)}{U(s)}=\frac{\frac{\beta\left(V_{i}-V_{o}\right)}{L C}}{s^{2}+\frac{1}{R_{L} C} s}
\end{gathered}
$$

where $Y(s)=X_{1}(s)=\beta V_{0}(s)$. Under the assumption that control signal is constant during the sampling period 
$T, u(t)=u(k T), k T<(k+1) T$, the discrete model in state-space form:

$$
\boldsymbol{x}(k+1)=\Phi \boldsymbol{x}(k)+\gamma u(k)+\boldsymbol{h}(k) .
$$

With assumption that no external disturbance is affecting on the system under control we have:

$$
\begin{aligned}
& \boldsymbol{x}(k+1)=\Phi \boldsymbol{x}(k)+\gamma u(k) \\
& y(k)=\boldsymbol{C} \boldsymbol{x}(k)
\end{aligned}
$$

where:

$$
\Phi=\mathrm{e}^{A T}
$$

Let:

$$
a=\frac{1}{R_{L} C}, b=\frac{\beta\left(V_{i}-V_{o}\right)}{L C} \text { so } \boldsymbol{A}=\left[\begin{array}{cc}
0 & 1 \\
0 & -a
\end{array}\right], \boldsymbol{B}=\left[\begin{array}{l}
0 \\
b
\end{array}\right]
$$

We get that:

$$
\begin{gathered}
\Phi=\left[\begin{array}{cc}
1 & \frac{1}{a}\left(1-\mathrm{e}^{-a T}\right) \\
0 & \mathrm{e}^{-a T}
\end{array}\right] \\
\gamma=\left(\int_{0}^{T} \Phi(\tau) \mathrm{d} \tau\right) * \boldsymbol{B} \\
\gamma=\left[\begin{array}{c}
\frac{b}{a}\left(T+\frac{1}{a}\left(\mathrm{e}^{-a T}-1\right)\right) \\
-\frac{b}{a}\left(\mathrm{e}^{-a T}-1\right)
\end{array}\right] .
\end{gathered}
$$

The model of the system with one input and one output is obtained from (3) and given in z-domain by the form:

$$
y(k)=\frac{z^{-1} B\left(z^{-1}\right)}{A\left(z^{-1}\right)} u(k)+\frac{z^{-1} \boldsymbol{D}\left(z^{-1}\right)}{A\left(z^{-1}\right)} \boldsymbol{h}(k) .
$$

As it is before proposed that system under control has no external disturbances affecting on it, so the control law:

$$
y(k)=\frac{z^{-1} B\left(z^{-1}\right)}{A\left(z^{-1}\right)} u(k) .
$$

The polynomials $A\left(z^{-1}\right), B\left(z^{-1}\right)$ can be found through:

$$
\begin{aligned}
& A\left(z^{-1}\right)=z^{-1} \operatorname{det}(z \boldsymbol{I}-\boldsymbol{\Phi}) \\
& B\left(z^{-1}\right)=z^{-n+1} \boldsymbol{C}[\operatorname{adj}(z \boldsymbol{I}-\boldsymbol{\Phi}) \boldsymbol{\gamma}] .
\end{aligned}
$$

And can be written as:

$$
\begin{aligned}
& A\left(z^{-1}\right)=a_{0}+a_{1} z^{-1}+\cdots+a_{n} z^{-n} \\
& B\left(z^{-1}\right)=b_{0}+b_{1} z^{-1}+\cdots+b_{m} z^{-m}
\end{aligned}
$$

where $n$-order of the system $(n=2), m$-number of inputs $(m=1), z^{-1}$ is the unit delay i.e. $z^{-1}=\mathrm{e}^{-p T}$, where $p$ denotes a complex variable. Using equation (12), we get: 


$$
\begin{aligned}
& a_{0}=1, \quad a_{1}=-\left(1+\mathrm{e}^{-a T}\right), \quad a_{2}=\mathrm{e}^{-a T}, \\
& b_{0}=\frac{b}{a} T+\frac{b}{a^{2}}\left(\mathrm{e}^{-a T}-1\right), \quad b_{1}=-\frac{b}{a} T \mathrm{e}^{-a T}-2 \frac{b}{a^{2}} \mathrm{e}^{-2 a T}+3 \frac{b}{a^{2}} \mathrm{e}^{-a T}-\frac{b}{a^{2}} .
\end{aligned}
$$

The goal is to maintain the sensed output voltage $y(k)=B v_{0}(k)$ stable, constant and equal to some constant reference voltage $V_{r}(k)=V_{\text {ref }}$, despite the variations of load resistance $R_{L}$ and input voltage $V_{i}$.

\subsection{Controller Design Procedure}

In order to get a better system steady state accuracy and chattering alleviation, the QSM (Quasi Sliding Mode) based on generalized minimum variance control (GMVC) technique is used, which is the combination of the discrete-time sliding mode and the generalized minimum variance control technique [11] [12].

Using the model of one input-one output given in (10), the design goal is to find a control law $u(k)$ that will force switching function to a minimum value (in the ideal case zero value) so that will keep the system motion in the vicinity of sliding surface $s(k)=0$, determined by the QSM domain $S$. Quasi-sliding mode is the movement along a predefined Near switching hypersurface $s(k)=0$, such that when one system trajectory enters this area never leaves it so that the relation $|s(k)|<\Delta$ always is valid, where $2 \Delta$ denotes the width of the area. The combination of digital QSM control and GMV control, was aimed so each of these components enhance the advantageous properties of the other. The general minimum variance GMV allow the digital QSMC to be realized only by the measurements of the output as well as to simplify the application of the QSM controlling of system which its zeros are on the unit circle. Also, the GMV control takes place as equivalent control in this type of digital QSMC, while digital QSMC was introduced with the aim to improve the robustness of GMV control when external disturbances and parameter perturbations are active [11].

The switching function in this case is:

$$
s(k+1)=C\left(z^{-1}\right)\left(y(k+1)-V_{r}(k+1)\right)+Q\left(z^{-1}\right) u(k) .
$$

In our case we have:

$$
C\left(z^{-1}\right)=c_{0}+c_{1}\left(z^{-1}\right)+c_{2}\left(z^{-2}\right)
$$

The polynomial $C\left(z^{-1}\right)$ can be calculated by:

$$
C\left(z^{-1}\right)=\left(1-\mathrm{e}^{-2 \pi f_{c} T} z^{-1}\right)^{2}
$$

where $C\left(z^{-1}\right)$ is a polynomial with all zeros inside the unit circle, while the polynomial $Q\left(z^{-1}\right)$ must satisfy the following equality in the steady state, i.e. where $z \rightarrow 1$ :

$$
Q(1)=0 .
$$

Control law, which fulfills the requirements, is the GMVC which is determined by:

$$
u(k)=-\frac{F\left(z^{-1}\right) y(k)-C\left(z^{-1}\right) V_{r}(k+1)}{E\left(z^{-1}\right) B\left(z^{-1}\right)+Q\left(z^{-1}\right)}
$$

where $E\left(z^{-1}\right)$ and $F\left(z^{-1}\right)$ are polynomials obtained as the solutions of Diophantine equation, using the equations (12), (15) we get the polynomials $E\left(z^{-1}\right)$ and $F\left(z^{-1}\right)$ of Diophantine equation:

$$
\begin{gathered}
c\left(z^{-1}\right)=E\left(z^{-1}\right) A_{n}\left(z^{-1}\right)+z^{-1} F\left(z^{-1}\right) \\
E\left(z^{-1}\right)=e_{0}=\frac{c_{0}}{a_{0}^{n}}, \quad F\left(z^{-1}\right)=f_{0}+f_{1} z^{-1} \\
f_{0}=c_{1}-e_{0} a_{1}^{n}, \quad f_{1}=c_{2}-e_{0} a_{2}^{n} .
\end{gathered}
$$

Theorem 1. For a system described in (10), the necessary and sufficient condition for the system with GMVC 
to be stable when all roots of the equation:

$$
B\left(z^{-1}\right) C\left(z^{-1}\right)+A\left(z^{-1}\right) Q\left(z^{-1}\right)=0
$$

are inside the unit circle in the $z$-plane, and the pairs $\left(B\left(z^{-1}\right), Q\left(z^{-1}\right)\right),\left(C\left(z^{-1}\right), A\left(z^{-1}\right)\right)$ and $\left(C\left(z^{-1}\right), Q\left(z^{-1}\right)\right)$ do not have a common zero outside this circle.

Proof: When the system under control is described by Equation (10), Equation (14) can be written as:

$$
y(k)=V_{r}(k)+\frac{1}{C\left(z^{-1}\right)} s(k)-\frac{Q\left(z^{-1}\right)}{C\left(z^{-1}\right)} u(k-1) .
$$

Substituting control law $u(k-1)$ from (10) in the last equation we have:

$$
y(k)=V_{r}(k)+\frac{1}{C\left(z^{-1}\right)} s(k)-\frac{Q\left(z^{-1}\right) A\left(z^{-1}\right)}{B\left(z^{-1}\right) C\left(z^{-1}\right)} y(k)+\frac{Q\left(z^{-1}\right) D\left(z^{-1}\right)}{B\left(z^{-1}\right) C\left(z^{-1}\right)} h(k-1) .
$$

Rearranging last equation, we get the final expression for the dynamics of the system in a closed loop:

$$
\begin{aligned}
y(k)= & \frac{B\left(z^{-1}\right) C\left(z^{-1}\right)}{B\left(z^{-1}\right) C\left(z^{-1}\right)+A\left(z^{-1}\right) Q\left(z^{-1}\right)} V_{r}(k)+\frac{B\left(z^{-1}\right)}{B\left(z^{-1}\right) C\left(z^{-1}\right)+A\left(z^{-1}\right) Q\left(z^{-1}\right)} s(k) \\
& -\frac{Q\left(z^{-1}\right) D\left(z^{-1}\right)}{B\left(z^{-1}\right) C\left(z^{-1}\right)+A\left(z^{-1}\right) Q\left(z^{-1}\right)} h(k-1) .
\end{aligned}
$$

The condition for stability of the closed-loop will be fulfilled if the expression in the denominator $B\left(z^{-1}\right) C\left(z^{-1}\right)$ $+A\left(z^{-1}\right) Q\left(z^{-1}\right)$ is stable, i.e. the roots of the polynomial lies inside the unit circle in $z$-domain. The request of couples do not have common zeros outside the unit circle arises from the need to avoid shortening of unstable roots, which cause instability in the system in case of parametric perturbations. As the dynamics of the closed loop of the system is derived based on the model of the system, and expression for variable $s(k)$ (which in case of digital QSMC takes place as switching function), the requirements which should be fulfilled in order to achieve stability in the closed loop system, and which are given in Theorem 1, will be identical for all variants of GMVC, including the digitally GMVC with QSM. Rewriting expression (25) in order to analyze the accuracy of the system:

$$
\begin{aligned}
& y(k)=\frac{B\left(z^{-1}\right) C\left(z^{-1}\right)}{1+\frac{A\left(z^{-1}\right) Q\left(z^{-1}\right)}{B\left(z^{-1}\right) C\left(z^{-1}\right)}} V_{r}(k)+\frac{B\left(z^{-1}\right)}{1+\frac{A\left(z^{-1}\right) Q\left(z^{-1}\right)}{B\left(z^{-1}\right) C\left(z^{-1}\right)}} \frac{1}{c\left(z^{-1}\right)} s(k) \\
& -\frac{Q\left(z^{-1}\right) D\left(z^{-1}\right)}{B\left(z^{-1}\right) C\left(z^{-1}\right)+A\left(z^{-1}\right) Q\left(z^{-1}\right)} h(k-1) \text {. }
\end{aligned}
$$

It is obvious that the dynamics of the system with GMV control leads to MV control when $Q\left(z^{-1}\right)=0$, Thus, at steady state ( $k \rightarrow \infty$ and $z \rightarrow 1$ ), and on the basis of (17) we have:

$$
\left.\frac{A\left(z^{-1}\right) Q\left(z^{-1}\right)}{B\left(z^{-1}\right) C\left(z^{-1}\right)}\right|_{z \rightarrow 1}=0
$$

Respectively the output of the system $y(k)$ will follow the reference signal $V_{r}(k)$ with a precision that would depends on the accuracy of variables $s(k), Q\left(z^{-1}\right)$ is selected to be approximately equal to the reciprocal of the z-transformation of the disturbance, which is in our case is negligible. GMV control represents the substitution for the equivalent control $u_{e q}$ in QSM control, whose design is based on the model of the system in state space form, while the functions $s(k)$, whose minimum variance, i.e. zero in the deterministic case, attempt to achieve GMV control, takes place as the switching function in the digital QSM. The relationship between the model (7) and (10), or between the matrix and the system coefficient model input-output data with: 


$$
\begin{gathered}
{\left[\begin{array}{lllll}
a_{n} & a_{n-1} & \cdots & a_{1} & 1
\end{array}\right]\left[\begin{array}{c}
c \\
c \Phi \\
\vdots \\
c \Phi^{n-1} \\
c \Phi^{n}
\end{array}\right]=0} \\
b_{1}=c \gamma \\
b_{2}=\left[\begin{array}{ll}
a_{1} & 1
\end{array}\right]\left[\begin{array}{c}
c \\
c \Phi
\end{array}\right] \gamma \\
b_{3}=\left[\begin{array}{lll}
a_{2} & a_{1} & 1
\end{array}\right]\left[\begin{array}{c}
c \\
c \Phi \\
c \Phi^{2}
\end{array}\right] \gamma
\end{gathered}
$$

In the traditional digital QSMC sliding hypersurface is defined by the formula:

$$
s(k+1)=\boldsymbol{\sigma} \boldsymbol{x}(k+1)=0 .
$$

While the digital equivalent control, which shall ensure that the $s(k+1)=0$, is defined by:

$$
u_{e q}(k)=-(\sigma \gamma)^{-1} \sigma \Phi x(k), \quad \sigma \gamma \neq \mathbf{0} .
$$

In order to make a connection between the digital equivalent control $u_{e q}$ and GMV control, it is necessary to introduce a switching function $s(k+1)$ using the output $y_{k}$ and control signal $u_{k}$ as:

$$
\begin{gathered}
s(k+1)=F\left(z^{-1}\right) y(k)+G\left(z^{-1}\right) u(k) \\
F\left(z^{-1}\right)=f_{0}+f_{1} z^{-1}+f_{2} z^{-2}+\cdots+f_{n-1} z^{-n+1} \\
G\left(z^{-1}\right)=g_{0}+g_{1} z^{-1}+g_{2} z^{-2}+\cdots+g_{m-1} z^{-m+1} .
\end{gathered}
$$

Control action which provides $s(k+1)=0$ in this case, by virtue of (34), with:

$$
u(k)=-\frac{F\left(z^{-1}\right)}{C\left(z^{-1}\right)} y(k) .
$$

The relations between the coefficients of the polynomial $F\left(z^{-1}\right)$ and $G\left(z^{-1}\right)$, and space-state model (7) and (32) are presented in the following form:

$$
\begin{gathered}
{\left[\begin{array}{lllll}
f_{n-1} & f_{n-2} & \cdots & f_{0} & 1
\end{array}\right]\left[\begin{array}{c}
c \\
c \Phi \\
\vdots \\
c \Phi^{n-1} \\
\sigma \Phi^{n}
\end{array}\right]=0} \\
g_{0}=\sigma \gamma \\
g_{1}=\left[\begin{array}{ll}
f_{0} & 1
\end{array}\right]\left[\begin{array}{c}
c \\
\sigma \Phi
\end{array}\right] \gamma \\
g_{2}=\left[\begin{array}{lll}
f_{1} & f_{0} & 1
\end{array}\right]\left[\begin{array}{c}
c \\
c \Phi \\
\sigma \Phi^{2}
\end{array}\right] \gamma
\end{gathered}
$$


So the appropriate GMV control in case of autonomous systems can be presented as:

$$
u(k)=-\frac{F\left(z^{-1}\right)}{E\left(z^{-1}\right) B\left(z^{-1}\right)+Q\left(z^{-1}\right)} y(k) .
$$

By comparing the expression of control (37) and (43) we get:

$$
G\left(z^{-1}\right)=E\left(z^{-1}\right) B\left(z^{-1}\right)+Q\left(z^{-1}\right) .
$$

The choice of $s(k+1)$ in the form (34) is equivalent to the choice of (14), which we have made in the formation of GMV control assuming that (44) is fulfilled. So, we have:

$$
\begin{aligned}
\left.s(k+1)\right|_{V_{r}=0} & =z^{-1} F\left(z^{-1}\right) y(k+1)+E\left(z^{-1}\right) B\left(z^{-1}\right)+Q\left(z^{-1}\right) u(k) \\
& =\left(c\left(z^{-1}\right)-E\left(z^{-1}\right) A\left(z^{-1}\right)\right) * y(k+1)+\left(E\left(z^{-1}\right) B\left(z^{-1}\right)+Q\left(z^{-1}\right)\right) u(k) \\
& =c\left(z^{-1}\right) y(k+1)+Q\left(z^{-1}\right) u(k) .
\end{aligned}
$$

As we see that GMV control is the substitution for the equivalent control in QSM, whose design is based on the model in the space state form, while the functions $s(k)$, whose minimum variance, or its zero value in the deterministic case, we tried to achieve that GMV control, takes the place of the switching function in the digital QSM control. If the reference input signal in subsequent time points $\left(V_{r}(k+1), V_{r}(k+2), \cdots\right)$ is known, we can construct the control law for digital QSM based on GMV:

$$
u(k)=-\frac{F\left(z^{-1}\right) y(k)-C\left(z^{-1}\right) V_{r}(k+1)+\frac{\alpha T}{1-z^{-1}} \operatorname{sgn}(s(k))}{E\left(z^{-1}\right) B\left(z^{-1}\right)+Q\left(z^{-1}\right)}
$$

where $s(k)$ is defined by (14), is called switching the digital QSM control based on GMV, while the polynomials $E\left(z^{-1}\right)$ and $F\left(z^{-1}\right)$ solutions Diophantine Equations (20), (21), while the polynomial $Q\left(z^{-1}\right)$ satisfies the equality (17). Substituting control law (46) in the model of the system (7), taking into account the expressions (14) and (19), rearranging these equations we get:

$$
s(k+1)=C\left(z^{-1}\right)\left(y(k+1)-V_{r}(k+1)\right)+Q\left(z^{-1}\right) u(k)=-\frac{\alpha T}{1-z^{-1}} \operatorname{sgn}(s(k))+E\left(z^{-1}\right) \boldsymbol{D}\left(z^{-1}\right) \boldsymbol{h}\left(z^{-1}\right) .
$$

Or in other words:

$$
s(k+1)=s(k)-\alpha T \operatorname{sgn}(s(k))+E\left(z^{-1}\right) \boldsymbol{D}\left(z^{-1}\right) *(\boldsymbol{h}(k)-\boldsymbol{h}(k-1)) .
$$

Expression (48) determines the dynamics of the switching function when control law (46) is applied to the system (7). The control law (46) is just like the one in QSM control with MV which its accuracy $O\left(T^{2}\right)$ of the system (7).

Theorem 2. For the discrete system which is described by (7) and (46), where the switching function is given by (14), and its dynamics is determined by (48). If the parameter $\alpha$ chosen to satisfy the following inequality:

$$
\alpha T>\max \mid E\left(z^{-1}\right) \boldsymbol{D}\left(z^{-1}\right)(\boldsymbol{h}(k)-\boldsymbol{h}(k-1)) .
$$

Then there is a natural number $K_{0}=K_{0}\left(s_{0}\right)$, such that for all $k>K_{0}$ exists quasi-sliding mode in the area of $S_{1}$ $\left(T^{2}\right)$ which is defined by:

where $\alpha=O T$.

$$
S\left(T^{2}\right)=\left\{s_{k}:\left|s_{k}\right|\langle\alpha T+\alpha T\rangle \max \left|E\left(z^{-1}\right) \boldsymbol{D}\left(z^{-1}\right)(\boldsymbol{h}(k)-\boldsymbol{h}(k-1))\right|\right\}
$$

Consequence: The system described by (7) and (46) is stable if and only if:

1) In it there is a quasi-sliding mode, that is filled with inequality (49) for each $k$, and

2) The polynomial $C\left(z^{-1}\right)$ has all its roots inside the unit circle in the $z$-plane.

The stability of the system with the proposed control is achieved if the requirements are met with the follow- 
ing theorem:

Theorem 3. The system which is described by equations (7) and (46) is stable if:

1) Parameter $\alpha$ is chosen according to the Theorem 2, and

2) The conditions of Theorem 1 is achieved.

The accuracy of the system in steady state will be in $O\left(T^{2}\right)$ limits.

As a conclusion by choosing the value of the parameter $\alpha$ to satisfy (46), then according to Theorem 2 the quasi-sliding mode exist in the domain $S$, and consequently the output $y(k)$ will converge to the reference voltage $V_{\text {ref }}$ only in case that the polynomial $C\left(z^{-1}\right)$ is stable.

\section{Simulation and Discussion}

In the earliest work [3], is considered the converter with the conventional PWM controllers and the PWM-based SM voltage controller, here on this work is considered the digital PWM controller to illustrate the difference in performance between them.

In order to the implement the MATLAB-Simulink environment to simulate the digital circuit of the controller, a table of the Components is used to verify the DC-DC converter with the proposed quasi-sliding mode voltage controller and they are given in Table 1, to validate the design methodology, different values of input voltages, output resistance are taken to ensure the functionalities of the system based on generalized minimum variance method with the use of quasi-sliding mode. The following values are achieved to fulfill the simulation needs: sampling time $T=50 \mu \mathrm{s}$, switching gain $\alpha=200$, sensor gain $\beta=0.166, V_{\text {ref }}=8 \mathrm{v}$, respectively.

The Simulink block diagram representing close loop Scheme of Boost converter is given in Figure 2, from this circuit we conclude the following results for the case where the input voltage fluctuate:

\begin{tabular}{ccc} 
Table 1. Boost converter parameter values. & & \\
\hline Description & Symbol & Nominal value \\
\hline Input voltage & $V_{i}$ & $20 \mathrm{v}, 24 \mathrm{v}, 28 \mathrm{v}$ \\
Capacitance & $C$ & $230 \mu \mathrm{F}$ \\
Inductance & $L$ & $200 \mu \mathrm{H}$ \\
Switching frequency & $f_{s}$ & $200 \mathrm{Khz}$ \\
Inductor resistance & $r_{L}$ & $0.14 \Omega$ \\
Capacitance ESR & $r_{c}$ & $0.69 \mathrm{~m} \Omega$ \\
Average load resistance & $R_{L}$ & $48 \Omega$ \\
Minimum load resistance & $R_{L_{-} \min }$ & $24 \Omega$ \\
Maximum load resistance & $R_{L \_\max }$ & $240 \Omega$ \\
Desired output voltage & $V_{0}$ & $48 \mathrm{v}$ \\
\hline
\end{tabular}

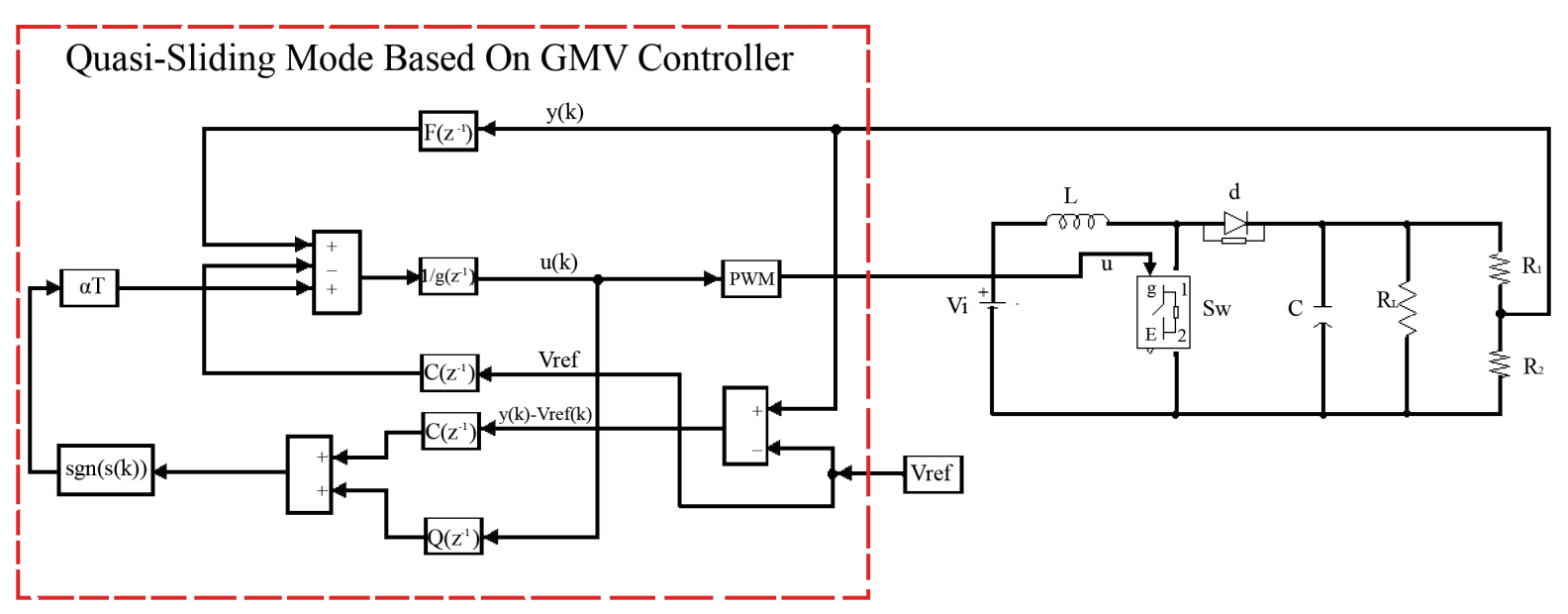


1) For $V_{i}=20 \mathrm{v}$ :

$A_{1}\left(z^{-1}\right)=1.0000-1.9955 z^{-1}+0.9955 z^{-2}$

$B_{1}\left(z^{-1}\right)=0.1266+0.1264 z^{-1}$

$C_{1}\left(z^{-1}\right)=1.0000-1.0670 z^{-1}+0.2846 z^{-2}$

Roots: $-0.7425,0.5914,0.4339$

2) For $V_{i}=24 \mathrm{v}$ :

$A_{2}\left(z^{-1}\right)=1.0000-1.9955 z^{-1}+0.9955 z^{-2}$

$B_{2}\left(z^{-1}\right)=0.1085+0.1084 z^{-1}$

$C_{2}\left(z^{-1}\right)=1.0000-1.0670 z^{-1}+0.2846 z^{-2}$

Roots: $-0.7017,0.5951,0.4220$

3) For $V_{i}=28 \mathrm{v}$ :

$A_{1}\left(z^{-1}\right)=1.0000-1.9955 z^{-1}+0.9955 z^{-2}$

$B_{1}\left(z^{-1}\right)=0.0904+0.0903 z^{-1}$

$C_{1}\left(z^{-1}\right)=1.0000-1.0670 z^{-1}+0.2846 z^{-2}$

Roots: $-0.6451,0.5997,0.4053$

The variation of the input voltage, and the load resistance are illustrated in Figure 3 and Figure 4.

Form the above figures we notice that when $R_{L}$ has its maximum value $240 \Omega$ the proposed quasi-sliding mode voltage controller provides robust Boost converter output voltage response, but the minimum value of the $R_{L}$ causes significant output oscillation about the reference input $V_{\text {ref }}$ but still gives the desired output voltage, also we notice that the uncertainties in DC-DC converter such as inductance and its resistance, current load resistance and uncontrollable input voltage It has been overcome using this technique with taking in mind that some uncertainties can be neglected such as the diode and switch voltage drop if the input voltage much greater than these drop voltages.

\section{Conclusion}

In this paper, we proposed the discrete-time quasi-sliding mode control strategies based on the so-called generalized minimum variance approach. This method guarantees improved robustness, faster transient response, and better steady-state accuracy of the controlled system than previously proposed algorithms of the same type such
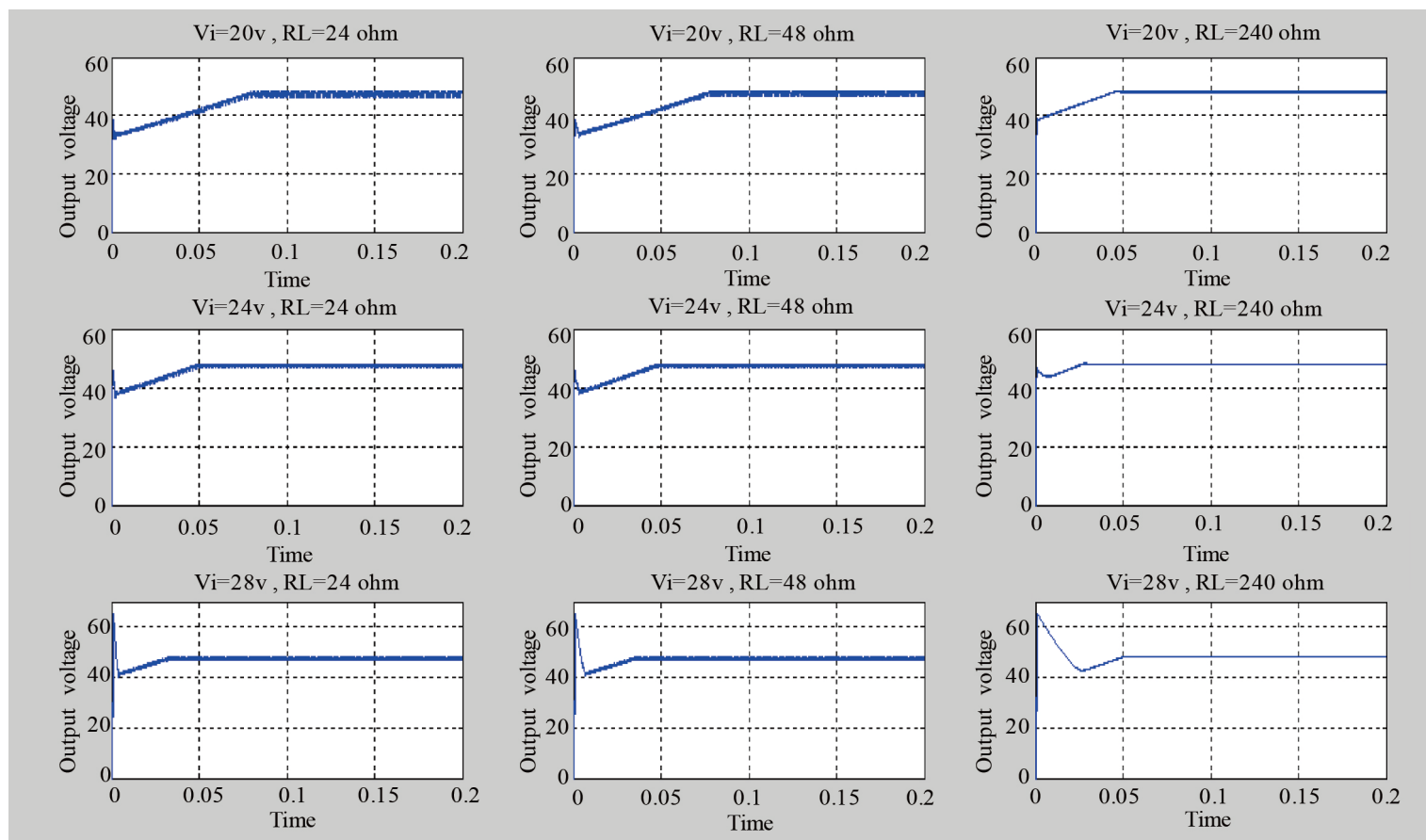

Figure 3. Waveform of the output voltage of the Boost DC-DC converter using QSMC based on GMV through the input voltage, and load resistance variation. 

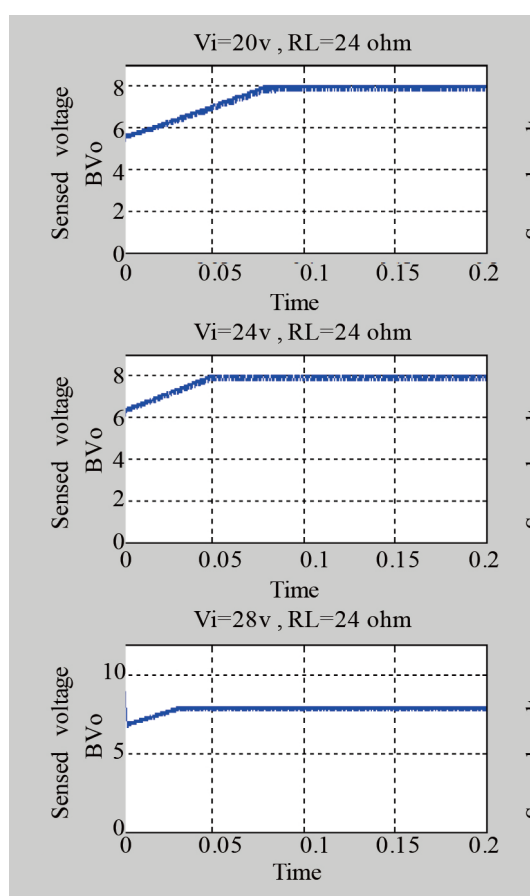

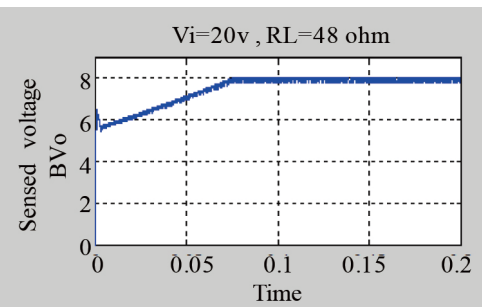

$\mathrm{Vi}=24 \mathrm{v}, \mathrm{RL}=48 \mathrm{ohm}$

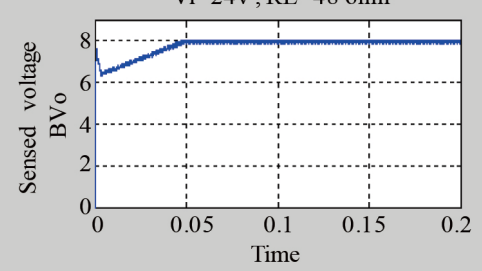

$\mathrm{Vi}=28 \mathrm{v}, \mathrm{RL}=48 \mathrm{ohm}$

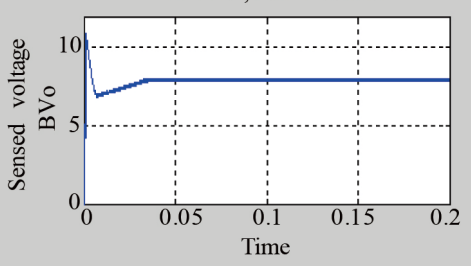

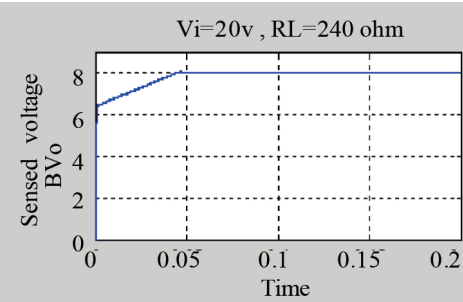
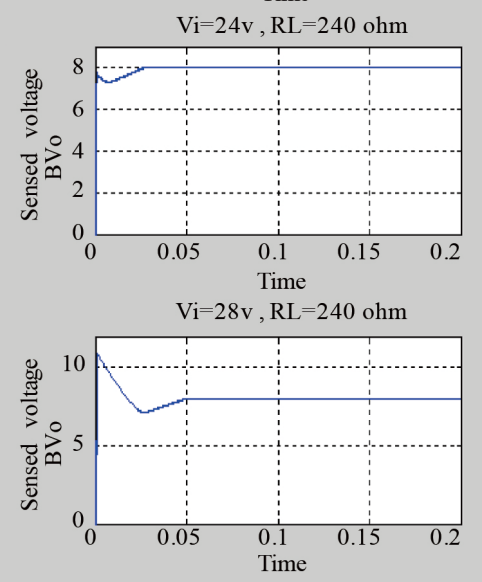

Figure 4. Waveform of the sensed output voltage of the Boost DC-DC converter using QSMC based on GMV through the input voltage, and load resistance variation.

as in [3]. In our definition, the conditions of the quasi-sliding mode are relaxed. i.e., we require the system state to remain in a band around the sliding hyperplane, but not to cross the hyperplane in each successive control step. Consequently, we proposed quasi-sliding-mode control strategies without chattering and high-frequency switching of the control signal. The better performance of our strategies is verified both theoretically and by simulation. The sensed output voltage $\left(\beta V_{0}\right)$ which is measured at the output (see Figure 2$)$ is compared with reference voltage $V_{\text {ref }}$ in order to get zero error voltage; this is held by using method GMV with the QSM which ensures the desired system requirements during parameter variations, gaining high output voltage accuracy in the presence of parameters perturbations; while the PWM is performed for switching the DC-DC Boost converter, this operation (controlling the duty cycle) is held by a train of pulses at the junction of $S_{W}$; the switching process is used as a replacement for the linear regulators when higher efficiency, smaller size and lighter weight are required. As a result, a stable output voltage is achieved in the presence of parameters perturbations.

\section{References}

[1] Erickson, R.W. and Maksimovic, D. (2000) Fundamentals of Power Electronics. 2nd Edition, Kluwer Academic Publishers, Dordrecht.

[2] Johansson, B. (2004) DC-DC Converters Dynamic Model Design and Experimental Verification. Ph.D. Dissertation, Lund University, Lund. http://lup.lub.lu.se/luur/download?func=downloadFile\&recordOId=24326\&fileOId=26469

[3] Tang, S.C., Lai, Y.M. and Tsi, C.K. (2006) A Unified Approach to the Design of PWM-Based Sliding-Mode Voltage Controllers for Basic DC-DC Converters in Continuous Conduction Mode. IEEE Transactions on Circuits and Systems, 53, 1816-1826. http://dx.doi.org/10.1109/TCSI.2006.879052

[4] Utkin, V.I. (1978) Sliding Modes and Their Applications in Variable Structure Systems. Moscow, Nauka, Mir.

[5] Mattavelli, P., Rossetto, L., Spiazzi, G. and Tenti, P. (1993) General-Purpose Sliding-Mode Controller for DC/DC Converter Applications. IEEE Power Electronics Specialists Conference Record (PESC), Seattle, 20 June-24 June 1993, 609-615. http://dx.doi.org/10.1109/pesc.1993.471989

[6] Venkataramanan, R. (1986) Sliding Mode Control of Power Converters. Ph.D. Dissertation, California Institute of Technology, Pasadena.

[7] Milosavljević, Č. (1985) General Conditions of the Existence of a Quasi-Sliding Mode on the Switching Hyperplane in Discrete Variable Structure Systems. Automatic Remote Control, 46, 307-314. 
[8] Mitić, D. and Milosavljević, Č. (2004) Sliding Mode Based Minimum Variance and Generalized Minimum Variance Controls with $O\left(T^{2}\right)$ and $O\left(T^{3}\right)$ Accuracy. Journal of Electrical Engineering (Archiv fur Elektrotechnik), 86, 229-237.

[9] Sira-Ramirez, H.J. and Ilic, M. (1988) A Geometric Approach to the Feedback Control of Switch Mode DC-to-DC Power Supplies. IEEE Transactions on Circuit and Systems, 35, 1291-1298. http://dx.doi.org/10.1109/31.7602

[10] Mitić, D. (2006) Digital Variable Structure Systems Based on Input-Output Model. Ph.D. Disertation, University of Niš, Niš.

[11] Mitic, D. and Antic, D. (2012) Input-Output Based Quasi-Sliding Mode Control of DC-DC Converter. Facta Universities, Series: Electronics and Energetic, 25, 69-80. http://dx.doi.org/10.2298/FUEE1201069M

[12] Tang, S.C. (2008) General Design Issues of Sliding-Mode Controllers in DC-DC Converters. IEEE Proceeding of Transactions on Circuits and Systems, 55, 1160-1174.

[13] Morel, C., Guignard, C. and Guillet, M. (2002) Sliding Mode Control of DC-DC Power Converters. Proceedings of the 9th International Conference of Electronics, Circuits Systems, 3, 971-974. http://dx.doi.org/10.1109/ICECS.2002.1046411

[14] Vazquez, N., Hernandez, C., Alvarez, J. and Arau, J. (2003) Sliding Mode Control for DC/DC Converters: A New Sliding Surface. Proceedings of the IEEE International Symposium on Industrial Electronics, 1, 422-426. http://dx.doi.org/10.1109/isie.2003.1267286 